\title{
Highly optimised global organisation of metabolic networks
}

\author{
R. Tanaka, M. Csete and J. Doyle
}

\begin{abstract}
High-level, mathematically precise descriptions of the global organisation of complex metabolic networks are necessary for understanding the global structure of metabolic networks, the interpretation and integration of large amounts of biologic data (sequences, various -omics) and ultimately for rational design of therapies for disease processes. Metabolic networks are highly organised to execute their function efficiently while tolerating wide variation in their environment. These networks are constrained by physical requirements (e.g. conservation of energy, redox and small moieties) but are also remarkably robust and evolvable. The authors use well-known features of the stoichiometry of bacterial metabolic networks to demonstrate how network architecture facilitates such capabilities, and to develop a minimal abstract metabolism which incorporates the known features of the stoichiometry and respects the constraints on enzymes and reactions. This model shows that the essential functionality and constraints drive the tradeoffs between robustness and fragility, as well as the large-scale structure and organisation of the whole network, particularly high variability. The authors emphasise how domainspecific constraints and tradeoffs imposed by the environment are important factors in shaping stoichiometry. Importantly, the consequence of these highly organised tradeoffs and tolerances is an architecture that has a highly structured modularity that is self-dissimilar and scale-rich.
\end{abstract}

\section{Introduction}

Metabolic networks, which have been extensively studied for decades, are emblematic of how evolution has sculpted biologic systems for optimal function. In addition to unambiguous functional descriptions of core metabolism, this conserved network has been recently described in detail in terms of its stoichiometry (mass and energy balance). A higher level, mathematically defined description of the global organisation of complex metabolic networks is critical for a deep understanding of metabolism, from the interpretation of huge amounts of biologic data (sequences, various -omics) to design of therapies for disease processes. The stakes are high for obtaining the big picture right: biologic data plugged into a distorted model or interpreted in the context of a flawed universal law propagates misinterpretations.

In flux analyses [1], stoichiometry is considered as a constraint, and fluxes are optimised to satisfy a global objective, typically growth. Previous studies, however, have not directly addressed whether the stoichiometry itself is highly optimal or organised in any sense and contributes to the origins and purpose of complexity in biological networks. Yet biochemistry textbooks describe metabolism as having evolved to be 'highly integrated' with the appearance of a 'coherent design' [2]. Here we

\section{(C) IEE, 2005}

IEE Proceedings online no. 20050042

doi:10.1049/ip-syb:20050042

Paper first received 1st July and in revised form 7th October 2005

R. Tanaka is with RIKEN, Bio-Mimetic Control Research Center, Nagoya 463-0003, Japan

M. Csete is with Emory University, Atlanta, GA 30322, USA

J. Doyle is with California Institute of Technology, Pasadena, CA 91125, USA

E-mail: reiko@bmc.riken.jp explore both important 'design' (with no implication of a 'designer') features of metabolism and the sense in which stoichiometry itself has highly organised and optimised tolerances and tradeoffs (HOT) [3] for functional requirements such as flexibility, efficiency, robustness and evolvability, constrained by conservation of energy, redox and small moieties.

This paper illustrates these features using the wellunderstood stoichiometry of metabolic networks in bacteria and reviews, compares and extends the results in $[4,5]$. We then propose a simple HOT model of an abstract metabolism to clarify the essential elements of its architecture. These features are not apparent from analyses that ignore organisation and constraints but rather use 'generic' ensemble properties, such as the popular 'scale-free' (SF) approaches [6]. Here we show that domain-specific constraints and tradeoffs imposed by the environment are important factors in stoichiometry. One consequence of this HOT architecture is a highly structured modularity that is self-dissimilar and scale-rich (SR).

\section{Basic features of metabolic networks}

Metabolism is essentially a linked series of chemical reactions, which function to synthesise building blocks for usable cellular components and to extract energy and reducing power from the cellular environment, in the context of total organism homeostasis. Constraints on the network are imposed by highly unpredictable intracellular and extracellular environments as well as the details of enzyme molecular structure, the cost of making enzymes and the conservation of atoms, energy and small moieties. The simplest model of metabolic networks is a stoichiometry matrix ( $s$-matrix for short) of chemical reactions with the metabolites in rows and reactions in columns and is defined unambiguously except for permutations of rows 
and columns. The simplest dynamic model of metabolism using an $s$-matrix $\boldsymbol{S}$ is given by $\mathrm{d} x / \mathrm{d} t=\boldsymbol{S} v(x)$, where $x$ is the concentrations of all the metabolites and $v(x)$ is the fluxes of all the reactions. We are temporarily deferring detailed consideration of $v(x)$ and focussing on just the properties of $\boldsymbol{S}$, although questions such as how the fluxes are distributed in the metabolic network and how these flux balances are regulated are of interest for deep understanding of metabolism. In this regard, flux balance analysis [1] has been successful in showing the flux balances that optimise growth rate at steady state given a fixed stoichiometry. We are, however, interested in how the structure and organisation of $\boldsymbol{S}$ facilitates control which is implemented via $v$ and in what sense the stoichiometry itself is optimal.

The appropriate arrangement of columns and rows based on biochemically meaningful categorisation clarifies the global structure of the $s$-matrix. Fig. 1 shows an $s$-matrix for Helicobacter pylori metabolism with the 'vertical' decomposition of reactions (columns) into standard functional modules, such as catabolism and biosynthesis, which are further split into amino acid, nucleotide, fatty acid/lipid/cell or cofactor biosynthesis, and the 'horizontal' decomposition of metabolites (rows) into carrier and non-carrier metabolites, which are further categorised into precursor and other (than precursor and carrier) metabolites (data from http://gcrg.ucsd.edu/ downloads). Catabolism transforms fuels into cellular energy and biosynthesis uses the energy for synthesis of amino acids, nucleotides, fatty acids/lipids/cell structures and cofactors. Carrier metabolites correspond to conserved quantities that are activated in catabolism and transfer energy by phosphate groups (ATP/ADP/AMP), hydrogen/ electrons (NADH/NAD), amino groups (AKG/GLU), acetyl groups (ACCOA/COA) and one carbon units (THF/ METHF) throughout all modules. As a result, carriers appear in many reactions. The standard 12 precursor metabolites (glucose 6-phosphate, fructose 6-phosphate, glyceraldehyde 3-phosphate, glycerone phosphate, 3-phosphoglycerate, phosphoenolpyruvate, pyruvate, ribose 5-phosphate, erythrose 4-phosphate, $\alpha$-ketoglutarate, oxaloacetate, and succinyl-CoA) are outputs of catabolism and are the starting points for biosynthesis.

By convention, carrier metabolites are in the bottom rows and enzyme intermediates are eliminated in the $s$-matrix. Categorisation of metabolites and reactions as in Fig. 1 clearly demonstrates the ubiquity of carriers and precursor

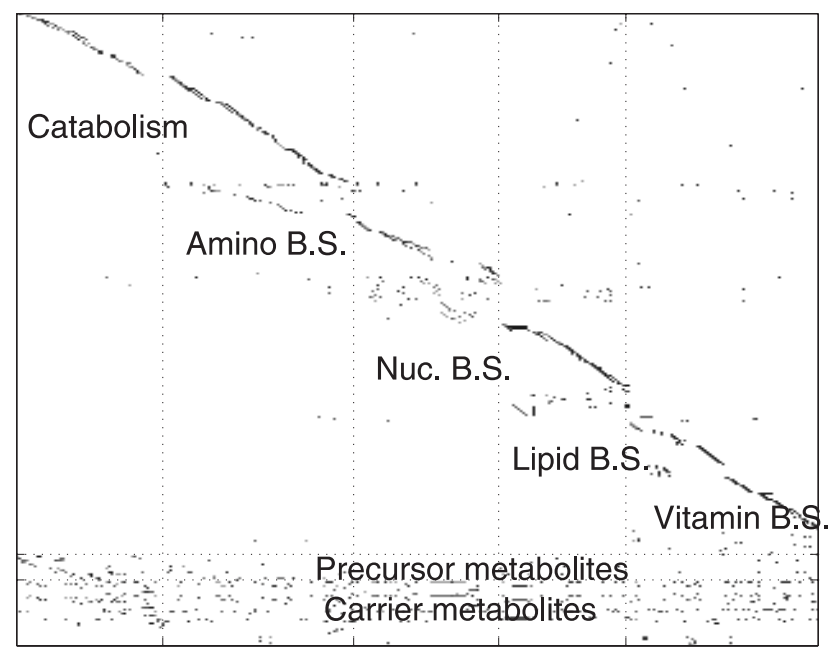

Fig. 1 S-matrix for $H$. Pylori metabolism with modular decompositions metabolites. The localised use of other metabolites in each reaction module consisting of long chains of reactions is shown by sparse diagonal elements in Fig. 1. This combination of extensive appearance of carriers and precursor metabolites in all reaction modules forming a 'knot', and the organisation of the remaining metabolites and reactions into long chains either feeding into or emanating from the knot, illustrates the 'bowtie' structure of metabolism (Fig. 3). Although $s$-matrices completely describe stoichiometry and this highly organised structure in principle, some features are still difficult to visualise. Graphs are popular and convenient ways to help visualise biological networks, but have also been a source of confusion when used improperly. Properly used, however, they can help visualise in more detail the implications of this bowtie architecture.

The information conveyed in the $s$-matrix can be faithfully represented and visualised in a colour-coded bipartite graph', called an $s$-graph [5], which is a minor variant of diagrams standard in biochemistry textbooks. Fig. 2 shows the $s$-graph for part of $H$. pylori metabolism, that is, part of glycolysis and the amino acid biosynthesis module of the $s$-matrix in Fig. 1. Both reactions and metabolites are represented as distinct nodes, and membership relationships of metabolites to reactions are represented by links. The metabolite nodes are further differentiated into those for carrier (blue) and non-carrier metabolites. Some carrier metabolites are always involved in reactions as a pair (ATP/ADP, NAD/NADH, etc.) and thus can be combined to simplify the $s$-graph. An $s$-graph thus consists of reaction nodes (black diamonds), carrier metabolite nodes (light blue squares) and non-carrier metabolite nodes (non-blue squares). Red and blue edges correspond to positive and negative elements in the $s$-matrix, respectively, for irreversible reactions, and pink and green ones correspond to positive and negative elements, respectively, for reversible reactions. With the colour-coding of links indicating the reversibility of reactions and the sign of elements in the $s$-matrix, all the biochemical information contained in the $s$-matrix is accurately reflected in the $s$-graph. Fig. 2 illustrates that long biosynthetic pathways build complex building blocks (in yellow on the right) from precursors (in orange on the left) in a series of simple reactions (in the middle), using shared common carriers (at the bottom). Each biosynthetic module has a qualitatively similar structure.

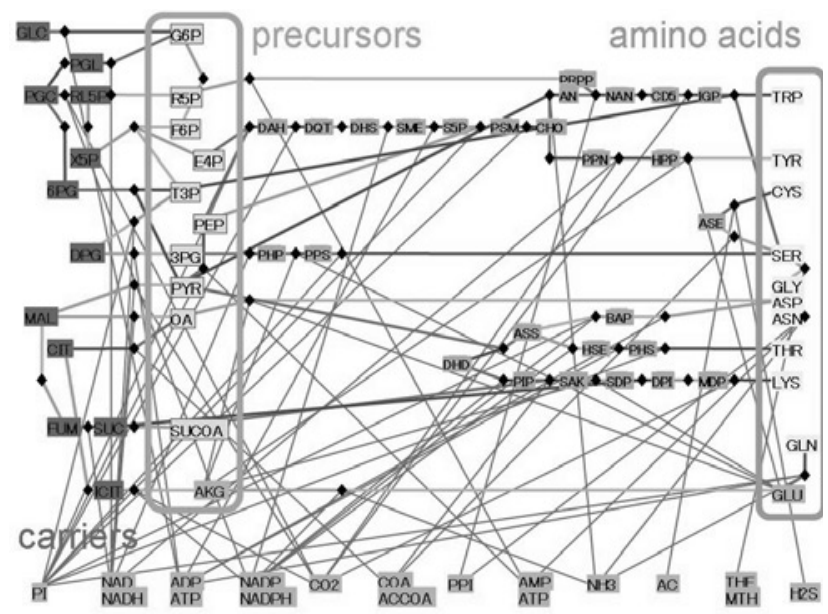

Fig. 2 An s-graph for part of glycolysis and the amino acid biosynthesis module for H.Pylori 
Combination of Figs. 1 and 2 is a good illustration of the 'bowtie' structure of metabolism [4], where a large 'fan-in' of nutrient inputs is catabolised to produce a small handful of activated carriers and precursor metabolites, which then 'fan-out' to the biosynthesis of a large number of primary building blocks (Fig. 3) through long chains of reactions. The bowtie structure of metabolism was independently derived from computational analyses [7]. The biologically natural modular decomposition of components is organised by the global structure into a 'knot' (of carriers and precursors) and non-'knot' parts of the 'bowtie'. The metabolism bowtie architecture and associated protocols allow highly optimised tradeoffs among multiple requirements. These include reaction complexity (number of metabolites in reaction), genome size and efficiency (energy required for each reaction). Of particular importance is the adaptability that is facilitated through tolerance of various perturbations, and flexibility and evolvability on longer time scales. This all occurs in the face of a large number of domain-specific constraints on conserved quantities [4]. In contrast, if every nutrient-product combination had independent pathways without shared precursors and carriers, the total genome would be vastly larger and/or enzymes would be vastly more complex. In either case, adaptation to fluctuating environments on any time scale would be difficult. Only an organisation like the bowtie facilitates the kind of extreme heterogeneity that allows for robust regulation, manageable genome sizes and biochemically plausible enzymes. Though not modelled explicitly in stoichiometry, this architecture facilitates the use of nested feedback interactions (protocols) to tightly regulate flux through the system, and these protocols are the source of both robustness and complexity of the network.

Although metabolism tolerates large fluctuations in nutrients and products, relatively small fluctuations in ATP are lethal. But the very architecture that creates this fragility also helps alleviate it, since ATP concentrations are tightly regulated and not easily changed. Another major source of fragility is that universal 'knot' responsible for robustness is essential, such that if it is hijacked or disrupted, then the entire network fails. Together, the efficiency and adaptability of metabolism along with its fragilities and power law degree distributions shown in the next section illustrate highly/heterogeneous optimised/organised tradeoffs/tolerance (HOT) [3] features. Quantitative evaluation of this HOT 'bowtie' architecture by biochemically realistic modular decomposition of metabolites and reactions reveals an SR topology of the network [5].

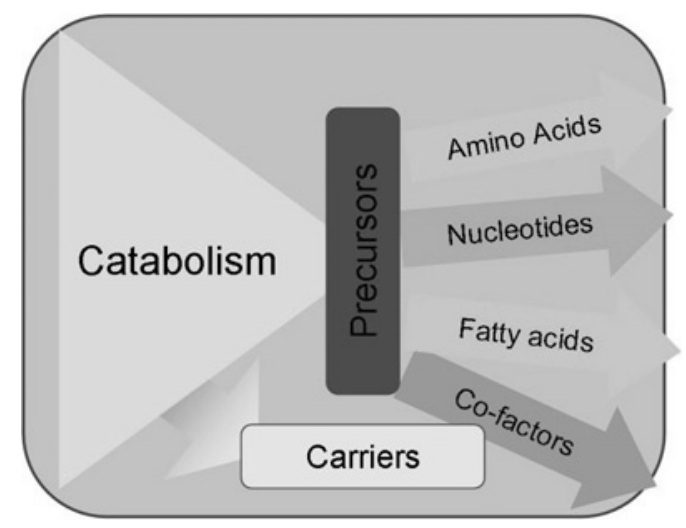

Fig. 3 Bowtie structure of core metabolism

\section{SR topology}

The topology of a network is a reflection of its architecture, which we define as a collection of protocols and their organisation. When topology is viewed in isolation, however, it can create misleading views of the system architecture. An example arises when considering certain network representations of stoichiometry where the distribution of metabolite 'node' degrees (number of reactions in which a metabolite is involved) satisfies a power law, and then this is taken as evidence that 'SF' networks underlie structure in biology [6]. In this regard, however, our analysis revealing SR organisation is supported by recent analysis [8] of protein-protein interaction networks that do not exhibit power law node degree distribution, and thus are not SF networks.

Although most of the graph-theoretical treatments of metabolic networks focus largely on the metabolite node degrees, it is important to note that degrees for both types of nodes, reaction and metabolite nodes, are biologically important (and equivalent to degrees of columns and rows, i.e. the number of non-zero elements in a column and in a row, of the $s$-matrix). In order to highlight the features of bacterial metabolism, the metabolite and reaction node degrees (the latter is the number of metabolites involved in a reaction) in $H$. pylori are shown in Fig. 4 $(+)$ and Fig. 5. It is plausible that the node degree for all metabolites follows an approximate power law. However, node degree sequence for reactions is not clearly described by a power law. It is thus important to understand the essential architectures responsible for generating these (very different) node degrees.

High variability (also called skewed or high dispersion of the data) is an even more fundamental characteristic of biologic networks than power laws. A standard measure of variability is the coefficient of variation $(\mathrm{CV}=\sigma / \mu$, where $\sigma$ and $\mu$ are sample standard deviation and mean). Exponential distributions have low variability and $\mathrm{CV}=1$, and power law distributions have divergent $\mathrm{CV}$ for large data sets (number of data $\rightarrow \infty$ ), thus high variability. For low variability processes, Gaussians arise naturally because of the well-known central limit theorem (CLT), and thus require no additional 'special' explanations. Even more important is that relaxing finite variance condition in the CLT yields power laws, which are further invariant under marginalisation, mixtures and maximisation [9]. Given the abundance of high variability phenomena and these strong invariants, power laws are an obvious null hypothesis and should properly be viewed as 'more normal than normal' [10]. Thus mechanisms responsible for high variability in

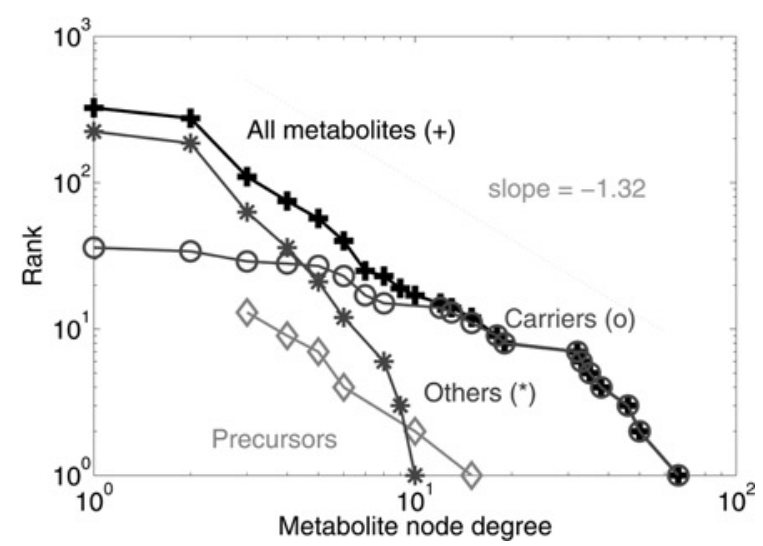

Fig. 4 Node degrees for carrier (०), precursor $(\diamond)$, other (*), and all (+) metabolites in H.Pylori metabolism 


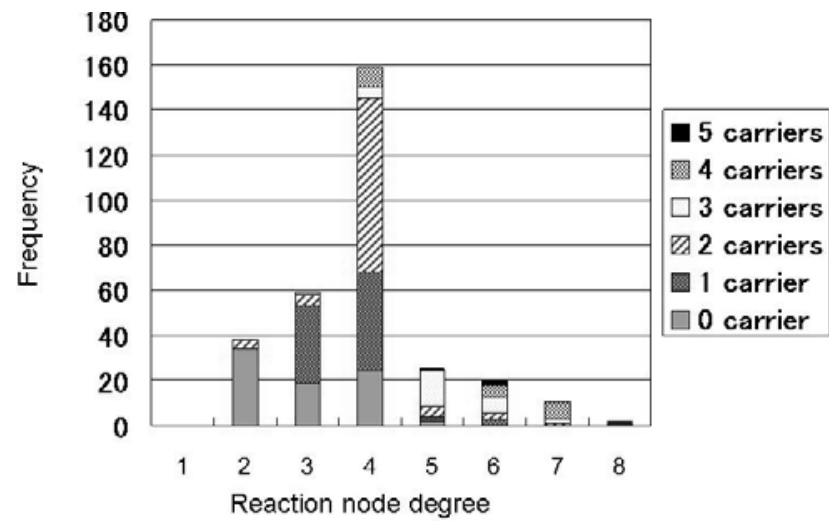

Fig. 5 Reaction node degree frequency for H.Pylori metabolism with contribution of carrier metabolites to degrees

total metabolite degrees are more fundamental in capturing the nature of biologic organisation than are power laws. We will argue here that high variability in metabolite degree and low variability in reaction degree are a necessary feature for any metabolic network to be efficient and robust to uncertain environments and cellular demands, although constrained by the physical limitations imposed on their chemical components.

Table 1 shows the CV and mean $\mu$ for the horizontal and vertical decompositions of the $s$-matrix in Fig. 1. Table 1 shows that the only place high variability appears is for all metabolites in the full network. It is obvious from the decomposition of metabolites into carrier, precursor and other metabolites that the high variability in the whole network is mainly created by high $\sigma$ from carrier metabolites mixed with low $\mu$ from others. This can also be seen in Fig. 4. The high $\sigma$ of all carrier metabolites comes from summing shared carrier metabolites across all the different reaction modules. Other metabolites appear almost uniquely in each reaction module and thus have both low $\mu$ and $\sigma$. Their sum across different modules has little effect on their degrees, and thus results in low degrees in aggregate. Decomposition of all metabolites in the full network into reaction modules also shows that total metabolites in each of the reaction modules have relatively low variability.

Table 1: CV and means of carrier, precursor, other, and all metabolite node degree distributions in catabolism (C) and amino acid (A), nucleotide (N), lipid (L), and vitamin (V) biosynthetic modules

\begin{tabular}{lllllll}
\hline & C & A & N & L & V & $\begin{array}{l}\text { All } \\
\text { reactions }\end{array}$ \\
& & & & & & \\
CV & & & & & & \\
Others & 0.38 & 0.49 & 0.56 & 0.67 & 0.42 & 0.61 \\
Precursors & 0.47 & 1.05 & 0 & 0.35 & 0.61 & 0.60 \\
Carriers & 0.50 & 0.81 & 1.23 & 0.64 & 0.92 & 1.13 \\
All metabolites & 0.63 & 0.88 & 1.20 & 0.90 & 1.04 & 1.72 \\
Mean $\mu$ & & & & & & \\
Others & & & & & & \\
Precursors & 3.38 & 1.56 & 1.00 & 1.25 & 2.00 & 5.54 \\
Carriers & 4.41 & 4.19 & 5.17 & 6.31 & 5.32 & 13.94 \\
All metabolites & 2.97 & 2.39 & 2.89 & 2.91 & 2.56 & 3.81 \\
\hline
\end{tabular}

Therefore the metabolic network is highly 'selfdissimilar' in the sense that the metabolite node degree distributions are very different at the full systems level (power law) and at all the module levels (exponentials), exactly opposite from the self-similarity described in SF networks. Metabolism consists of widely different scales in modules, and thus would more appropriately be called SR. Medium-degree precursors act as cores and high-degree carriers are directly related to many distinct uses of metabolic byproducts. Another important feature of metabolism derived from bowtie structure is that deletion of high-degree carriers does not fragment the network as is assumed for 'hubs' in the SF models, as carriers play an essential biochemical role in most reactions yet are not responsible for the long chains of reactions that connect the other metabolites and precursors. Removing the carriers from biologically meaningful $s$-graph in Fig. 2 still yields a fully connected network with long pathways between the remaining metabolites. In contrast, deleting precursor metabolites of only medium degree does (lethally) break up the pathways. Thus the true fragility of the network is obfuscated by focussing only on graph-theoretical properties of the network. It is possible to partially 'fix' this problem by a priori eliminating the carriers from graphs, but then the resulting graphs have low variability in metabolite node degree sequence and thus no longer have power laws, the defining feature of SF networks. Recent experimental results in [11] further shows that the degree of the metabolite nodes is not necessarily correlated with its lethality.

As shown above, the high variability comes from the bowtie structure of metabolism with a small knot of high degree common currencies (carriers and precursors). Its robustness facilitates control, accommodating perturbations and fluctuations on many time and spatial scales. That is, the $s$-matrix by itself does not explicitly represent the regulatory mechanisms which directly affect the fluxes, but the structure and organisation of the $s$-matrix is such that it greatly facilitates that regulation, and the high variability is its necessary side product. This analysis of the $s$-matrix suggests that the statistical features of metabolism, and particularly the presence of high variability and power laws, depend on its highly organised (HOT) structure for efficiency, robustness, and so on.

Very low variability in reaction node degrees as in Fig. 5 can be explained by standard biochemistry, as the enzymes of core metabolism are highly efficient and specialised for high fluxes of small metabolites and thus necessarily have few metabolites; they execute simple reactions resulting in long assembly chains. Thus, low variability in reaction node degrees results from the highly efficient, specialised enzymes in core metabolism. Note that the number of carriers involved in a reaction is also an important statistic as shown in Fig. 5, and a typical reaction has four metabolites of which two are carriers.

\section{$4 \quad$ HOT models}

The next analysis focusses on identifying the minimal features of metabolism responsible for the various structural features discussed above, including both high and low variability in metabolite and reaction node degrees, respectively, and long assembly chains. To this end, we will explore a highly simplified model that nevertheless realistically captures these particular features of metabolism. The aim is to go beyond simply characterising the structure of metabolic networks and determine the essential necessary functional requirements and component constraints leading to that structure. Very simple models of metabolism 
can reveal the essential constraints that drive HOT networks, and generating the simplest possible models to illustrate basic features of real metabolism is a natural starting point to which additional data and complexity can be added. These models must abstract both the biological function of metabolism and the constraints on its components.

We previously proposed a simple abstract model for metabolism [5] that is minimal, in both the biological sense that no simpler reactions are possible and the mathematical sense that it has minimal assumptions and can easily be solved analytically, yet reproduces the important characteristics of metabolic networks. This simplest model shows that the existence of universal carriers is by itself necessary and sufficient to have high and low variability in metabolite and reaction node degrees. Although the model explains what is minimally needed to produce the high $\mathrm{CV}$ in metabolite degree, it fails to capture other significant structural features of metabolism, in particular the long tree-like pathways of biosynthesis. We thus propose a slightly more complex HOT stoichiometry model, which we call the HOTword model, that contains long chains of pathways to synthesise building blocks, in addition to the high and low variability in metabolite and reaction node degrees.

The constraint on our abstract toy metabolites in HOTword model is the assumption that each 'metabolite' is described by a word such as 'ABCC' with each letter corresponding to a conserved quantity and its carrier (such as methyl or amino groups, phosphate, etc.). The constraints on reactions are that they can simply delete or add the last letter of the word, such as $\mathrm{ABCC} \leftarrow \rightarrow \mathrm{ABC}+\mathrm{C}$, and are all fully reversible. There are no separate precursors, as the single letter 'carriers' play both roles. These assumptions are very simplified abstractions of observations that most reactions in metabolism involve transfer of groups (such as phosphate or methyl groups or hydrogen) and that most reactions involve two non-carrier metabolites and one or a pair of carriers as shown in Fig. 5. We assume that a set of complex (word) metabolites requires no distinction between inputs and outputs since all reactions are reversible. Then we can define a minimal notion of robustness for a network in the sense that, with appropriate fluxes, it can realise any arbitrary stoichiometrically balanced set of reactions involving these I/O (input/ output) metabolites. Note that this extent of reversibility and identical inputs and outputs are not realistic assumptions but simplify the model and are easily removed.

Although it may not be immediately apparent, HOTword model is so simple that it is actually trivial to construct by hand a network which is 'optimal' or nearly so, and this was an additional objective, beyond its biologically motivated features. Specifically, an 'optimal' network here is a minimal tree network that is maximally robust and efficient in the sense of having the fewest possible number of reactions and intermediate metabolites while achieving perfect robustness to any arbitrary but stoichiometrically meaningful I/O metabolites and reactions. Although it is possible to define a formal algorithm to convert any list of I/O metabolites into an optimal network, a sketch of how this can be done using a simple example should, we hope, suffice to illustrate how such a process would work. Fig. 6 shows an s-graph visualisation of a network with four I/O metabolites consisting of two carriers ' $\mathrm{A}$ ' and 'B': one of length four 'BAAA'; two of length three, 'BAB' and 'BBA' and one of length two letters, 'BB'. Note that since each reaction node uses only one carrier, the $s$-graph can be simplified without loss of information by labelling the reaction (enzyme) node with its carrier and suppressing the carrier nodes.

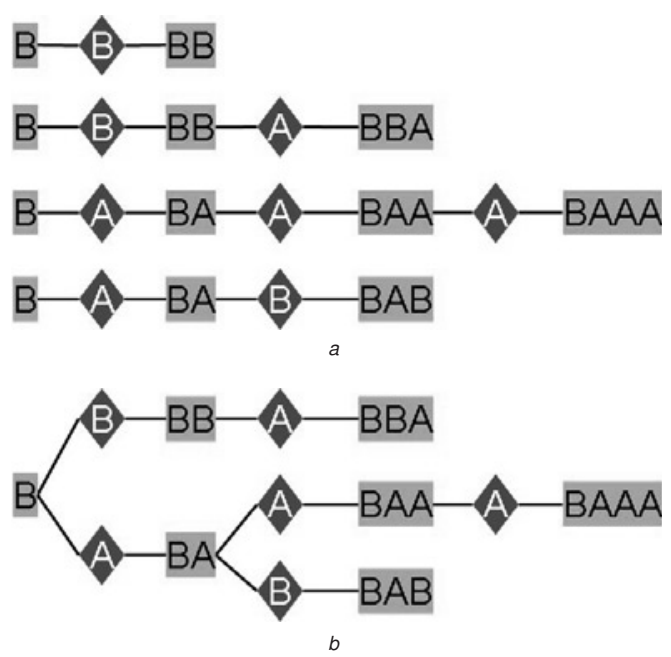

Fig. 6 An example of a HOTword model network. Rectangles and diamonds denote metabolite nodes and reaction nodes, respectively

A reaction node is labelled with its used carrier

$a$ A list of long chains of reactions with a letter on the left end and an $\mathrm{I} / \mathrm{O}$ metabolite on the right end

$b$ A functionally equivalent but minimal tree-structural graph obtained by merging common reactions in the chains

A simple $s$-graph tree is easily constructed by inspection, and the process can be illustrated using the model in Fig. 6. First, each I/O metabolite, such as 'BAAA', is reduced to its leftmost letter, here ' $\mathrm{B}$ ' via the intermediates ' $\mathrm{BA}$ ' and 'BAA'. This yields a list of long chains with a letter on one end and an I/O metabolite on the other as shown in Fig. $6 a$. This collection of simple reactions is obviously capable of collectively producing any stoichiometrically balanced reaction, but is not minimal as reactions may be repeated in different chains. These can be collapsed and trimmed into a tree of reactions as shown in Fig. $6 b$ by removing redundant reactions between chains. The resulting tree-like $s$-graph of reactions is minimal in having the fewest number of reactions that can still realise any complex, stoichiometrically balanced reaction. A rigorous proof, though straightforward, requires additional definitions and intermediate results, and is deferred here, in favour of exploring the biologically relevant consequences of the resulting structure.

Since all reactions are reversible, Fig. $6 b$ can be thought of as half of the canonical metabolism bowtie structure. The other half corresponding to the reconstruction of all $\mathrm{I} / \mathrm{O}$ metabolites from their components is given by exactly the same model run in reverse. (Clearly, the above construction can be extended to different inputs and outputs and irreversible reactions.) Thus this extremely simplified HOTword model fails to distinguish between catabolism and biosynthesis, but does capture many other features of real metabolism: (i) the constraint in conservation of small moieties, (ii) the typical low reaction degrees and the number of carriers involved and (iii) the functional requirements of conversion of nutrients to products by long tree-like pathways. An easy calculation shows that it always has a high variability metabolite node degree sequence created by a mixture of a heavy-tail consisting of carriers with low degree non-carriers. Accordingly, this HOTword model captures the minimal features of metabolism responsible for the high and low variability in metabolite and reaction node degree sequences, respectively, together with long assembly chains, that is, the features of bowtie structures. 


\section{Conclusions}

The HOT 'bowtie' structure is an important aspect of the highly optimised organisation and 'design' features of metabolism. It facilitates both great robustness and efficiency but is also a source of fragility [4]. A side-effect of this architecture is high variability in total metabolite node degree despite low variability in all metabolite and reaction modules. Approximate power laws are, statistically, almost inevitable given this high variability. We have shown that SR and highly dissimilar topology at every level of organisation, even when studied at just the level of stoichiometry, is a consequence of this HOT 'bowtie' structure of metabolism and is characterised by high and low variability in metabolite and reaction node degrees and long assembly chains of reactions. The regulation of enzyme levels by transcription, translation and degradation and enzyme activity by allostery and competitive inhibition adds additional richness to metabolic networks.

Following [5], in this paper, we proposed a simple HOT model of metabolism by abstracting the biological functionality of stoichiometry and the constraints on the enzymes and reactions of metabolism. It reveals what we claim are the essential functionality and constraints that drive the tradeoffs and hence the large-scale structure and organisation, particularly SR topologies. In contrast to approaches that treat metabolic networks as generic or random graphs, including those with power law degree distributions, this analysis of metabolic stoichiometry reveals a network that is highly organised and rather specific to metabolism or manufacturing. It arises out of the requirements for functionality of the network and for robustness and evolvability under realistic constraints.

\section{References}

1 Papin, J.A., Stelling, J., Price, N.D., Klamt, S., Schuster, S., and Palsson, B.O.: 'Comparison of network-based pathway analysis methods', Trends Biotechnol., 2004, 22, pp. 400-405

2 Berg, J.M., Tymoczko, J.L., and Stryer, L.: 'Biochemistry' (W. H. Freeman and Company, NY, 2002)

3 Carlson, J.M., and Doyle, J.C.: 'Complexity and robustness', Proc. Natl. Acad. Sci. USA, 2002, 99, (Suppl. 1), pp. 2538-2545

4 Csete, M., and Doyle, J.: 'Bowties, metabolism, and disease', Trends Biotechnol., 2004, 22, pp. 446-450

5 Tanaka, R.: 'Scale-rich metabolic networks', Phys. Rev. Lett., 2005, 94, p. 168101

6 Barabàsi, A.-L., and Oltvai, Z.N.: 'Network biology: understanding the cell's functional organisation', Nat. Rev. Genet., 2004, 5, pp. $101-113$

7 Ma, H., and Zeng, A.-P.: 'The connectivity structure, giant strong component and centrality of metabolic networks', Bioinformatics, 2003, 19, pp. 1423-1430

8 Tanaka, R., Yi, T.-M., and Doyle, J.: 'Some protein interaction data do not exhibit power law statistics', FEBS Lett., 2005, 579, pp. $5140-5144$

9 Mandelbrot, B.B.: 'Fractals and scaling in finance: discontinuity, concentration, risk' (Springer-Verlag, NY, 1997)

10 Willinger, W., Alderson, D., Doyle, J., and Li, L.: 'More 'normal' than normal: scaling distributions and complex systems'. Proc. 2004 Winter Simulation Conf., 2004

11 Mahadevan, R., and Palsson, B.O.: 'Properties of metabolic networks: structure versus function', Biophys. J.: Biophys. Lett., 2005, 88, L07-L09 